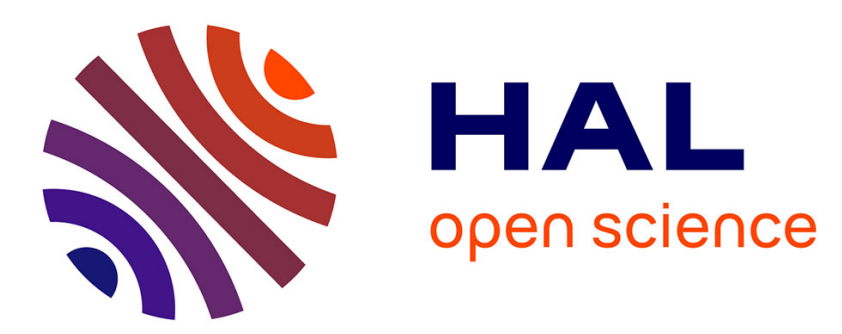

\title{
Detection and diagnosis of changes in the eigenstructure of nonstationary multivariable systems
}

Michèle Basseville, Albert Benveniste, George V. Moustakides, Anne Rougée

\section{To cite this version:}

Michèle Basseville, Albert Benveniste, George V. Moustakides, Anne Rougée. Detection and diagnosis of changes in the eigenstructure of nonstationary multivariable systems. [Research Report] RR-0477, INRIA. 1986. inria-00076077

\section{HAL Id: inria-00076077 https://hal.inria.fr/inria-00076077}

Submitted on 24 May 2006

HAL is a multi-disciplinary open access archive for the deposit and dissemination of scientific research documents, whether they are published or not. The documents may come from teaching and research institutions in France or abroad, or from public or private research centers.
L'archive ouverte pluridisciplinaire HAL, est destinée au dépôt et à la diffusion de documents scientifiques de niveau recherche, publiés ou non, émanant des établissements d'enseignement et de recherche français ou étrangers, des laboratoires publics ou privés. 


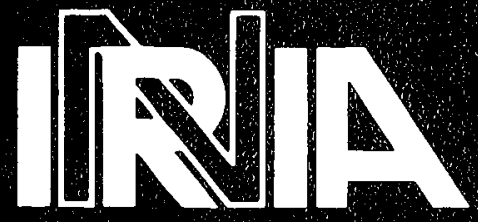

CENTRE DE RENNES

I RISA

\section{Rapports de Recherche}

$$
\mathrm{N}^{\circ} 477
$$

\section{DETECTION AND DIAGNOSIS OF CHANGES}

\section{IN THE EIGENSTRUCTURE OF NONSTATIONARY \\ MULTIVARIABLE SYSTEMS

OF CHANGES
IN THE EIGENSTRUCTURE
OF NONSTATIONARY
MULTIVARIABLE SYSTEMS

Instutut National de Recherche e. et en Automatique

Domaine de Volve eres

Roceviencoum 8.

\author{
Michèle BASSEVILLE \\ Albert BENVENISTE \\ Georges MOUSTAKIDES \\ Anne ROUGÉE
}

Janvier 1986 
Campus Universitaire de Beaulieu

35042-RENNES CÉDEX

FRANCE

Téléphone: 99362000

Télex: UNIRISA $950473 \mathrm{~F}$

Télécopie: 99383832

Publication Interne $n^{\circ} \quad 276$

Décembre 1985

44 pages

\section{DETECTION AND DIAGNOSIS OF CHANGES}

IN THE EIGENSTRUCTURE OF NONSTATIONARY MULTIVARIABLE SYSTEMS

Michèle Basseville [IRISA/CNRS], Albert BENVENISTE [IRISA/INRIA], Georges Moustakides [IRISA/INRIA], Anne Rougée [IRISA/Univ.]

Résumé.- On étudie les deux problèmes de détection et diagnostic de changements dans la matrice de transition d'état d'un système multivariable excité par un bruit d'état inconnu et non-stationnaire. On propose des tests nouveaux. dits "instrumentaux". dont on montre numériquement l'efficacité. même pour des changements petits. On décrit enfin l'application de ces méthodes au problème de surveillance des vibrations pour les plateformes of fshore.

Abstract.- The two problems of detection and diagnosis of changes in the state transition matrix of a multivariable system with nonstationary unknown state noise, are addressed. New instrumental tests are derived and shown to be numerically powerful, even for small changes. The application to vibration monitoring of offshore platforms is described.

Travail soutenu par le contrat IFREMER $n^{\circ}$ 84/7392 et le GRECO CNRS SARTA. 
PI 269 Modélisation et gestion d'univers 30 (une première approche a partir du logiciel PADL-2

Gérard Hégron, Patrick Rives - 60 pages ; Octobre 85.

PI 270 LGRC : Un langage pour la synthese d'images par lancer de rayons

Bruno Arnaldi, Kadi Bouatouch, Thierry Priol - 30 pages ; Novembre 85.

PI 271 Towards specification and proof of asynchronous systems Boubakar Gamatié - 30 pages ; Novembre 85.

PI 272 Architecture pour les opérations geométriques en synthèse d'images par facettes

François Charot, Franck Rousée - 24 pages ; Novembre 85.

PI 273 Madmacs : a new VLSI layout macro editor

Patrice Frison, Eric Gautrin - 12 pages ; Novembre 85.

PI 274 Détection de pannes et reconfiguration automatique

Michèle Basseville - 26 pages ; Novembre 85.

PI 275 Estimation de l'ordre d'un processus Arma à l'aide de résultats de perturbations de matrices

Jean-Jacques Fuchs - 40 pages ; Décembre 85.

\title{
DETECTION AND DIAGNOSIS OF CHANGE
}

IN THE EIGENSTRUCTURE

OF NONSTATIONARY

Detection and diagnosis of changes in the eigenstructure of nonstationary multivariable systems

Michèle Basseville, Albert Benveniste, Georges Moustakides,

Anne Rougée - 44 pages ; Décembre 85.

\author{
MULTIVARIABLE SYSTEMS
}

$$
n^{\circ} 276
$$




\section{DETECTION AND DIAGNDSIS OF CHANGES IN THE EIGENSTRUCTURE OF NONGTATIONARY MULTIVARIABLE SYSTEMS}

Michèle Basseville*, Albert Benveniste**, Georges Moustakides**. Anne Rougée

Abstract. - The two problems of detection and diagnosis of changes in the state transition matrix of a multivariable system with nonstationary unknown state noise, are addressed. New instrumental tests are derived and shown to be numerically powerful, even for small changes. The application to vibration monitoring of offshore platforms is described.

This work was supported by IFREMER grant $n^{\circ} 84 / 7392$ and by CNRS GRECO SARTA. The authors are with IRISA, Campus de Beaulieu, 35042 Rennes Cédex, France. *Also with CNRS

**AZso with INRIA 


\section{INTRODUCTION}

The problem of detecting changes in dynamical systems has received a large attention in Willsky (1976), Mironovski1 (1980), Pau (1981), Isermann (1984), M. Basseville, A. Benveniste (Ed.) (1986), in many fields of application, for the purposes of fallure detection in controlled systems or signal segmentation for recognition. Most of the time domain model-based methods use the entire set of the known or estimated model parameters for solving the two basic steps of change detection, namely residual generation and choice of the (statistical) decision function (Willsky, 1976). For example, both filter innovations and parity checks involve all the model parameters, with possible inclusion of parameters uncertainties, and on the other hand classical likelihood ratio or bayesian tests proceed similarly.

However, for some applications, it may be necessary to detect changes in one subset of the model parameters without knowing or using any estimates of the parameters belonging to the complementary subset. These last parameters have therefore to be considered as nuisance parameters. In section IV, we will describe such an application concerned with vibration monitoring of a structure subject to nonstationary and unmeasured natural excitation. The related change detection problem may be formulated in the two following equivalent ways :

i) consider a multivariable observable system described by the discrete time state space model :

$$
\left\{\begin{array}{l}
x_{t+1}=F x_{t}+V_{t+1}, \quad \operatorname{cov}\left(V_{t+1}\right)=Q_{t} \\
Y_{t}=H x_{t}
\end{array}\right.
$$


where the dimensions of the state $X$ and the observation $Y$ are $n$ and $r$ respectively, with $r$ (much) smaller than $n$ in practice, and where the additive state noise $v_{t}$ is an unmeasured gaussian process with timevarying covariance matrix $Q_{t}$. Then the problem is to detect changes in the state transition matrix $F$, up to a change of basis, without using the nuisance parameters $Q_{t}$ which may be highly time-varying (see section IV). Here the observation matrix $H$ is assumed to be fixed; the related problem of optimal sensor location for change detection will be reported elsewhere.

1i) consider a multidimensional ARMA process :

$$
Y_{t}=\sum_{i=1}^{p} A_{i} Y_{t-1}+\sum_{j=0}^{p-1} B_{j}(t) E_{t-j}
$$

with constant autoregressive $r \times r$ parameters $\left(A_{1}\right)_{1 \leqslant 1 \leqslant p}$ and time-varying moving average $r \times r$ parameters $\left(B_{j}\right)_{0 \leqslant j \leqslant P-1}$; and where $\left(E_{t}\right)_{t}$ is a gaussian white noise with identity covariance matrix. The model in (2) may be obtained from (1) in a classical way (Akaike, 1974) by solving the following linear system of equations :

$$
H F^{P}=\sum_{i=1}^{p} A_{i} H F^{p-i}
$$

Let $\theta^{\top}=\left(A_{p}, \ldots, A_{1}\right)(4)$ be the set of AR parameters.

In this situation, the problem is to detect changes in the AR parameters $\theta$ without using the nuisance moving-average parameters $B_{j}(t)(0 \leqslant j \leqslant p-1)$. 
In each of these two model formulations, the second problem we address here is the problem of dlagnosis : when, a change has been detected, decide which pole(s) and elgenvector(s) have been changed. In (1), we are interested in monitoring the eigenstructure of the system, 1.e. the pairs $\left(\lambda, H \phi_{\lambda}\right.$ ) where $\lambda$ and $\phi_{\lambda}$ are the elgenvalues and corresponding eigenvectors of the state transition matrix $F$, which is assumed to be asymptotically stable. In (2), we consider equivalently the generalized eigenvalues and eigenvectors of the matrix polynomial :

$$
I-\sum_{i=1}^{P} A_{1} z^{-1}
$$

In section IV, these will be the vibrating characteristics of the monitored structure.

Because of the timing characteristics of our application, namely very slowly occuring changes (months or years) and high sampling frequency, in all what follows, we will solve rather a model validation problem than a change detection problem : namely, given a reference model, $F^{0}$ or $\theta^{0}$ and. a new record of observations $\left[Y_{t}\right]_{1 \leqslant t \leqslant s}$, decide whether this model still adequately describe 1 ( (global detection) and solve the diagnosis problem. However, the tests which we propose here may be used for on-line change detection purposes, as generally explained in M.'Basseville, A. Benveniste (Ed.) (1986) : this has been done for speech signal segmentation in R. André-Obrecht (1985).

The paper is organized as follows. In section II, we present our original approach for solving the global detection problem, using both an 
instrumental statistics and a statistical local approach for detection. In section III, we study the diagnosis problem, following the same local approach and using a linearization to relate changes in eigencharacteristics to changes in AR parameters. Numerical experiments on both simulated and real data are reported in section $V$, following the description of the underlying mechanical engineering problem of vibration monitoring, which is given in section IV. Finally some conclusions are drawn in section VI.

\section{GLOBAL CHANGE DETECTION}

In this section, we investigate the two equivalent problems of detecting changes in the $F$ matrix of model (1) or in the AR parameters $\left(A_{i}\right)_{i}$ of

model (2), with as much robustness as possible with respect to the nonstationary unknown excitation $v_{t}$ or moving average parameters $\left(\theta_{j}(t)\right)_{j}$.

As discussed in Basseville et al. (1985b) in the scalar case, it is not possible to use standard likelihood techniques for solving this problem. The two key reasons are that, first, the Fisher information matrix of an ARMA process is not block-dlagonal, and thus there is a coupling effect between poles and zeroes; and, second, the highly time varying behavior of the unknown parameters prevent from using nuisance parameters elimination methods, usually based upon estimation or integration. We recall that in Bohlin (1977) for example, convenient estimated values of the MA coefficients were used.

The idea of our approach is to transform the problems ij or ii) into the simpler problem of detecting a change in the mean of a Gaussian process 
with known covariance matrix, which is then solved by the classical $\chi^{2}$ (genralized) likelihood ratio test.

\section{An instrumental statistics}

For the above mentionned purpose, we consider the following so-called instrumental statistics :

$$
U_{N}(s)=\sum_{t=1}^{s} z_{t} w_{t}^{\top}
$$

where :

$$
Z_{t}^{\top}=\left(Y_{t-p}^{\top}, \ldots, Y_{t-P-N+1}^{\top}\right)
$$

is a vector of $N \gtreqless p$ instrumental variables, i.e. here simply delayed observations, and where $W_{t}$ is

$$
W_{t}=Y_{t}-\sum_{i=1}^{P} A_{i}^{0} Y_{t-1}=Y_{t}-\theta^{\dot{O} T} \phi_{t}
$$

with

$$
\phi_{t}^{\top}=\left(Y_{t-p}^{\top}, \ldots, Y_{t-1}^{\top}\right)
$$

$\theta^{0}$ is the reference AR model, the adequacy of which has to be tested for the new record $\left(\gamma_{t}\right)_{1 \leqslant t \leqslant s}$. The process $U_{N}(s)$ may be alternatively generated in a practically more attractive way, using the following formula :

$$
U_{N}(s)=H_{p+1, N}^{T}(s)\left(\begin{array}{c}
\theta^{0} \\
-I_{r}
\end{array}\right)
$$

where $\mathcal{H}_{p+1, N}(s)$ is the empirical Hankel matrix of the observed process $\left(Y_{t}\right):$ 


$$
H_{p, q}(s)=\left(\begin{array}{lc}
\left.R_{0} s\right) \ldots & R_{q-1}(s) \\
\vdots & \vdots \\
R_{p-1}(s) \ldots R_{p+q-2}(s)
\end{array}\right), R_{m}(s)=\sum_{t=1}^{s-m} Y_{t+m} Y_{t}^{\top} .
$$

It is well known (Söderström and Stoica, 1980) that equating to zero the right-hand side of (7) and solving, in the least squares sense if $N>p$, the resulting system of delayed Yule-Walker equations is nothing but the instrumental variable I.V. identification method, the consistency of which is established under stationary conditions. Benveniste and Fuchs (1985) show that the I.V. method also leads to a consistent estimate of the AR parameters $\theta$, in the present situation of nonstationary unknown MA coefficients. This last robustness property is numerically proved in Prevosto et al. (1982), and in Prevosto et al. (1983) where favorable comparisons with frequency domain methods are made. Let us now investigate the detection problem for the process $U_{N}$.

Under the hypothesis $H_{0}$ of no change, ie $\theta=\theta^{0}, W_{t}$ defined by (5) is actually a MA process, which is uncorrelated with $z_{t}$, and thus $U_{N}(s)$ is zero-mean.

Under the local alternative hypothesis $H_{1}$ of small change, ie $\theta=\theta^{0}+\frac{\delta \theta}{\sqrt{s}}$, the mean of $U_{N}(s)$ can be easily checked to be equal to the mean of :

$$
\frac{1}{\sqrt{ } s} \mathscr{H}_{P, N}^{T}(s) \delta \theta
$$


In order to be able to compute covariances, it is necessary to consider a convenient vector $\mathcal{U}_{N}$ instead of the matrix $U_{N}$, and from now on we will use notations and basic results concerning kronecker products which have been summarized for example in Yuan and Ljung (1984).

Let :

$$
\begin{aligned}
U_{N}(s) & \triangleq \operatorname{col}\left(U_{N}^{\top}(s)\right) \\
& =\sum_{t=1}^{s} z_{t} \otimes w_{t}
\end{aligned}
$$

be the vector of size $\mathrm{Nr}^{2}$ obtained by stacking the $\mathrm{Nr}$ columns of $\mathrm{U}_{N}^{\top}(s)$ on top of each other, and $\Theta=\operatorname{col}\left(\theta^{\top}\right)$ obtained in the same way from $\theta$ (4). Furthermore, let $\sum_{N}(s)$ be the covariance matrix of $\boldsymbol{U}_{N}(s)$ under the no change hypothesis $H_{0}$. Then, because of the independance of $\left(Z_{t}, W_{t}\right)$ and $\left(Z_{s}, W_{s}\right)$ for $|t-s| \geqslant p$, we have :

$$
\Sigma_{N}(s)=\sum_{t=1}^{s} \sum_{i=-p+1}^{p-1} \quad \mathbb{E}_{0}\left(z_{t} z_{t-i}^{T} \otimes w_{t} w_{t-i}^{\top}\right)
$$

Let us consider the following estimate :

$$
\dot{\Sigma}_{N}(s)=\sum_{t=1}^{s} \sum_{i=-p+1}^{p-1}\left(z_{t} z_{t-i}^{\top} \otimes w_{t} w_{t-i}^{\top}\right)
$$

It is shown in Moustakides and Benveniste (1985), that $\frac{1}{s} \hat{\sum}_{N}(s)$ is a consistent estimate of $\frac{1}{s} \sum_{N}(s)$ under both hypotheses $H_{0}$ and $H_{1}$ (because first order approximations do not affect covariances), and that $\frac{1}{\sqrt{ }} U_{N}(s)$ is asymptotically gaussian distributed under both hypotheses, ie :

$$
\begin{aligned}
& \sum_{N}^{-1 / 2}(s) U_{N}(s) \frac{\mathcal{L}}{s+\infty} \mathcal{N}_{(0, I)} \text { under } H_{0} \\
& \sum_{N}^{-1 / 2}(s)\left(U_{N}(s)-\mu(s)\right) \frac{\mathscr{Q}}{s_{N \rightarrow \infty}} \mathcal{X}_{(0, I)} \text { under } H_{1}
\end{aligned}
$$


where

$$
\mu(s)=\frac{1}{\sqrt{s}}\left(\mathcal{H}_{P, N}^{T}(s) \otimes I_{r}\right) \delta \Theta
$$

Let us emphaslze that these law of large numbers and central limit theorems are obtained in the nonstationary framework we have assumed (time-varying moving average parameters). Therefore, as stated at the beginning of this section, we have transformed the initial problems i) and i1) into the classical problem of change in the mean of a gaussian process with known covariance matrix. Precise assumptions and theorem statements are given in Appendix A.

\section{The changes which can be detected}

We now emphasize which changes can be detected with the ald of the instrumental statistics $U_{N}(s)$ (7). Because of 1 ts mean value $(8)$ under $H_{1}, U_{N}(s)$ will allow the detection of any change $\delta \theta$ belonging to the range of $\frac{1}{s} H_{P, N}^{T}(s)$.

We assume that the nominal representation $\left(H_{0}, F_{0}\right)(1)$ is observable and that the following factorization of the empirical Hankel matrix holds :

$$
\frac{1}{s} \mathcal{H}_{P, N}(s)=\theta_{P}\left(H_{0}, F_{0}\right) \cdot \varphi_{N}\left(F_{0}, G_{s}\right)+\varepsilon(s)
$$

where

$$
\theta_{p}\left(H_{0}, F_{0}\right)=\left(\begin{array}{c}
H_{0} \\
H_{0} F_{0} \\
\vdots \\
H_{0} F_{0}{ }^{p-1}
\end{array}\right)
$$

is the observability matrix, and 


$$
\ell_{N}\left(F_{0}, G_{s}\right)=\left(G_{S}, F_{0} G_{S}, \ldots, F_{0}^{N-1} G_{S}\right)
$$

is the controllability matrix, uniformely of full row rank $n$, $G_{5}$ being the empirical cross-correlation between the state $X$ and the observation $Y$. In (12), $\varepsilon(s)$ converges to zero in distribution as sto. We refer to Appendix $A$ for the assumptions under which these properties are true.

In such a case, because of the factorization (12), the only changes on $\theta$ we will not be able to detect with this approach are those that satisfy :

$$
\theta_{p}^{T}\left(H_{0}, F_{0}\right) \delta \theta=0
$$

But these last changes do not correspond to any change in the minimal representation (1) of the system, as the following argument shows. In fact, formula (3) shows that the representations (1) and (2) of the system are related to each other through the relationship :

$$
\theta_{\mathrm{p}+1}^{\mathrm{T}}\left(\mathrm{H}_{0}, \mathrm{~F}_{0}\right) \quad \theta_{0}=0
$$

and any $\theta_{0}$ satisfying this relation gives raise to a valid ARMA representation of the system. But two different parameters $\theta_{0}$ and $\theta_{0}+\delta \theta$ satisfying the above relation, are precisely related through (13). Further discussions may be found in (Benveniste et al.) (1985). This property (13) is of particular importance in practice, because it means that, for detecting changes in a minimal representation $\left(H_{0}, F_{0}\right)(1)$ of $\left(Y_{t}\right)$ with 
the aid of the instrumental statistics $U_{N}(s)$ (7), it is not necessary to use a minimal ARMA representation (2) of $\left(Y_{t}\right)$.

\section{The global test}

We now give the test for detecting changes in the mean of $U_{N}(s)$ (9). Recall that, if a vector $U$ is gaussian with mean $\mu$ and covariance $\sum$, for testing $\mu=0$ against $\mu \in$ Range $(M)$ where $M$ is a full column rank matrix, the (generalized) likelihood ratio test is :

$$
U^{\top} \Sigma^{-1} M\left(M^{\top} \Sigma^{-1} \cdot M\right)^{-1} M^{\top} \Sigma^{-1} U
$$

Let us consider the application of this result to the instrumental statistics $\mathcal{U}_{N}(\mathrm{~s})$

In order to apply (14), it is necessary to reduce

$$
M=\mathscr{L}_{p, N} T(s) \otimes I_{r}
$$

to a full (nr) column rank matrix. This is possible because the system (1) is assumed to be observable. A solution involving selection of rows in $\theta_{p}\left(H_{0}, F_{0}\right)$ and of columns in $\mathscr{H}_{p, N}(s)$ is described in (Basseville et al... 1985a) and (Rougée, 1985).

Therefore, according to the previous discussion, the global test for detecting changes in $\Theta$ is :

$$
t_{0}=U_{N}^{\top}(s) \quad \dot{\xi}_{N}^{-1}(s) \quad M\left(M^{\top} \hat{\Sigma}_{N}^{-1}(s) M\right)^{-1} M^{\top} \dot{\Sigma}_{N}^{-1}(s) U_{N}(s)
$$


with $M$ given by (15). Under $H_{0}, t_{0}$ is distributed as a $\chi^{2}$ variable with $n r$ degrees of freedom. Under $H_{1}$, to is distributed as a $\chi^{\prime 2}$ with the same number of degrees of freedom, and with non centrality parameter :

$$
\delta \Theta M^{\top} \quad \dot{\Sigma}_{N}^{-1}(s) \quad M \quad \delta \Theta
$$

which is non zero in all the cases of interest as previously discussed.

Let us briefly mention an interesting special case which will be useful In practice. Assume that the numbers of sensors $r$ divides the state dimension $n$, that the AR order of the ARMA model (2) is $p=n / r$ (this means that the first $n$ rows of $\theta_{p}\left(H_{0}, F_{0}\right)$ are independent), and that the number of instruments $N$ is equal to the $A R$ order $p$. In this situation, $M$ (15) is Invertible and the global test reduces to :

$$
t_{0}=U_{p}^{\top}(s) \dot{\Sigma}_{p}^{-1}(s) U_{p}(s)
$$

The efficiency of the test $\{16\}$ is numerically investigated in section IV, and theoretically analyzed by Rougée (1985) and Rougée et al. (1985) under stationarity assumptions. It turns out that, in the case where $n=p r$, Ie where the dimension of the observation is a divisor of the state dimension, the asymptotic power of the test $t_{0}$ is related to the asymptotic precision of the Instrumental variable identification method, as derived by Stoica et al. (1985). Furthermore, it may be stiown that, in the scalar case and for a special cholce of instruments, this test is equivalent to the min-max optimal local likelihood test, which is robust with respect to uncertainties on the moving average part. Finally, preliminary numerical results for nonstationary scalar signals are described by Basseville et al. (1985b) and show that small changes $(1 \%)$ in eigenfrequencies may 
be detected, using sufficlently long records.

\section{THE DIAGNOSIS PROBLEM}

We now discuss the diagnosis problem, as stated in the introduction. When a change on the AR part has been detected by the global test $t_{0}$, the problem 1s to get insights onto which poles and modes have been changed, again without knowing the nonstationary MA part. As far as we known, the multiple model approach is the only solution which has ever been given to this problem, even in the stationary autoregressive case, ie when in (2) only $B_{0}$ is non zero and is moreover constant.

Our suggestion is to use the same approach as for the global test, together with a relationship between changes in eigencharacteristics and changes in AR parameters, to be used in the expression (11) of the mean value of the instrumental statistics $\mathcal{U}_{\mathrm{N}}$ under $H_{1}$. As we develop our detection tools under the assumption of small changes, we use first order Taylor expansions for $\Theta$, in the following manner.

Let $\Phi$ a minimal parameterization of the AR part of the process. In section IV, $\Phi$ will be the set of the eigen (or modal) characteristics of the structure. We assume that the application $\Phi \rightarrow \Theta=f(\Phi)$ is continuously differentiable in the neighborhood of the nominal model $\Phi_{0}$. If we want to monitor a particular subset of the coordinates of $\Phi$, we consider the matrix $f$ obtained by selection of the corresponding columns of the jacobian matrix $f^{\prime}\left(\Phi_{0}\right)$, and we apply formula (16) with :

$$
M=\left(\mathcal{H}_{P, N}^{T}(s) \otimes I_{r}\right) \quad \mathcal{J}
$$


in order to get what we call a sensitivity test.

Several computations of Jacobians of interest for our vibration monitoring application are described in section IV.

The main advantage of this method is to allow separate monitoring of subsets of parameters of interest (for example one pole together with the corresponding eigenvector), without knowing in advance which subsets will actually change. The main drawback is that no theoretical argument may assess some decoupling property, concerning for example separate manitoring of modes : the corresponding tests (16) computed with the relevant Jacobians (19) are not statistically independent of each other. However, simulations results presented by Basseville et al. (1985b) show that, in the scalar case $(r=1)$, this decoupling property concerning poles hold, provided that the poles are close to unit circle. In the vector case, this seems to be still true, provided that furthermore the number of sensors $r$ is equal to the actual number of modes $(n / 2$ in case of real $F$ ). See (Basseville, 1985).

Finally, let us mention another approach for the diagnosis problem which is investigated by Basseville et al. (1985b) in the sçalar case. This approach is based upon a theoretical decoupling property, but is difficult to be implemented because of combinatorial problems in (real) case of no a prior1 knowledge of which parameter subsets are to change. 


\section{APPLICATION TO THE VIBRATION MONITORING PROBLEM}

\section{Motivàtions}

As mentionned in the introduction, the reason for which we studied the problems addressed in this paper is a mechanical engineering problem: how to supervise the vibrating characteristics of a structure subject to a nonstationary and unmeasured natural excitation. Examples of such vibrating structures are offshore platforms subject to the swel I (the purpose of our study), buildings or bridges subject to wind or earthquakes, mechanical objects subject to fluid interactions,... One of the goals of vibration monitoring is the detection of cracks and fatigues. Dur approach for solving such a problem involves the following steps :

i. On site identification of the vibrating characteristics of the structure. This step is necessary because the finite elements models provided by the designer have to be significantly readjusted when the structure is settled down. Furthermore, the possible model deviations due to fatigue appearence are often less important than the deviations between the designer's model and the behavior actually observed when the structure is settled down. Therefore the designer's model cannot be used as a reference model for fatigue detection by modal analysis.

ii. Detection of changes in the modal characteristics. As explained in the Introduction, the purpose of this task is to decide whether the model which was identifled on the safe structure still adequately represent the new accelerometer signals obtained during a new inspection. 
iii. Diagnosis of the change. The problem is then to discriminate between changes in the masses distribution, which are of no interest for fatigue detection, and changes in the stiffness coefficients; and furthermore to estimate the localization of the fatigues in the structure.

From the implementation point of view, tasks 1 and $11 i$ should be done in a remote computing center because they are time consuming. Task il, previously called global detection in section II, should be done on board.

Up to our knowledge, it seems that no other parametric model approach has been followed for solving these types of problems. Furthermore the way by which we manage with the excitation and the fluid/structure interactions also seems to be non-standard. Because the vibrating modes of an offshore platform lie beyond one hertz (and thus beyond the fundamental frequency of the swell), the structure is mainly excited by shock effects and turbulences which induce non-stationarities in the signals and which are unmeasurable by the existing swell sensors. In such a situation, our approach is to model the excitation by a nonstationary gaussian white noise, to neglect the fluid/structure interactions, and to develop algorithms as much robust as possible with respect to these assumptions, for the three above mentionned tasks.

\section{The model to be used}

Assuming that the structure may be decomposed in finite elements and has a linear behavior (Prevosto, 1982), we model a vibrating structure by a damped system of masses connected by springs, which obeys the following equation :

$$
M \ddot{D}_{t}+C \dot{D}_{t}+K D_{t}=\varepsilon_{t}
$$


where $M, C, K$ are the masses, damping, and stiffness matrices respectively; $D_{t}$ is the vector of displacement of the $m$ degrees of freedom; $\varepsilon_{t}$ is the excitation vector, assumed to be a non-stationary gaussian white noise with covariance matrix $R_{t}$. The modal characteristics $\left(\lambda, \phi_{\lambda}\right)$ of this structure are the solutions of :

$$
\left\{\begin{array}{l}
\operatorname{det}\left(M \lambda^{2}+c \lambda+K\right)=0 \\
\left(M \lambda^{2}+C \lambda+K\right) \phi_{\lambda}=0
\end{array}\right.
$$

As M, C, $K$ are real, the $2 \mathrm{~m}$ solutions $\lambda=c+i \omega$ are pairwise conjugate. They are called eigenfrequencies; the $\omega$ are the eigen-pulsations; and $c / \sqrt{c^{2}+\omega^{2}}$ is the corresponding (negative) damping coefficient. In case of proportional dampling, ie when $C=\alpha M+\beta K$, the modes $\phi_{\lambda}$ are real.

Let $Y_{t}$ be the observation of $r \leqslant m$ degrees of freedom of the system. $Y_{t}$ is described by :

$$
Y_{t}=L D_{t}
$$

where $L_{i j}$ is equal to one of sensor $i$ observes the degree $j$, and zero otherwise. Then the modal identification is the obtention of $(\Delta, L \Phi)$ where $\Delta$ is the diagonal matrix filled by the $\lambda$, and $L \Phi$ is the observed part of the modes $\phi_{\lambda}$.

The system described by the equations (20) and (22) is equivalent to the following continuous time state space model : 
$-18-$

$$
\begin{aligned}
& \left\{\begin{array}{l}
\dot{x}_{t}=A x_{t}+B_{t} \\
y_{t}=H x_{t}
\end{array}\right. \\
& \text { where } x_{t}=\left(\begin{array}{c}
D_{t} \\
\dot{D}_{t}
\end{array}\right) \text {, dim } x_{t}=2 m=n, A=\left(\begin{array}{cc}
0 & I \\
-M^{-1} K & -M^{-1} c
\end{array}\right), B_{t}=\left(\begin{array}{c}
0 \\
-M^{-1} \varepsilon_{t}
\end{array}\right) \\
& H=\left(\begin{array}{ll}
L & 0
\end{array}\right) \text {. The discrete time equivalent model is: } \\
& \left\{\begin{array}{l}
x_{t+\Delta t}=F x_{t}+V_{t} \\
y_{t}=H x_{t}
\end{array}\right.
\end{aligned}
$$

Because of the above assumption on $\varepsilon_{t}(20), V_{t}$ is a white noise with covariance matrix :

where

$$
\begin{aligned}
& Q_{t}=\int_{t}^{t+\Delta t} \cdot e^{A \tau} \cdot \tilde{R}_{\tau} \cdot e^{A^{\top} \tau} d \tau \\
& \tilde{R}_{\tau}=\left(\begin{array}{cc}
0 & 0 \\
0 & M^{-1} R_{\tau} M^{-1}
\end{array}\right) .
\end{aligned}
$$

The eigenvalues $\mu$ and eigenvectors $\psi_{\mu}$ of $F(24)$ are related to the modal characteristics (21) by :

$$
\mu=e^{\lambda \cdot \Delta t} \quad \text { and } \quad H \psi_{\mu}=L_{\lambda}
$$


and are solutions of :

$$
\left(\mu^{P} I_{r}-\sum_{j=1}^{P} \mu^{P-j} A_{j}\right) H \psi_{\mu}=0
$$

where the autoregressive parameters $A_{1} \ldots, A_{p}$ are given by (3). Actualiy, assuming that $\Delta t=1, F$ may be diagonalized in the following manner:

$$
F=\Psi \cdot \theta^{D} \cdot \Psi^{-1}
$$

where

$$
\begin{aligned}
& \mathrm{D}=\left(\begin{array}{cc}
\Delta & 0 \\
0 & \bar{\Delta}
\end{array}\right) \text { and : } \\
& \Psi=\left(\begin{array}{cc}
\Phi & \bar{\Phi} \\
\Phi \Delta & \overline{\Phi \Delta}
\end{array}\right) \text {. }
\end{aligned}
$$

Consequently, identifying and monitoring the set of $\left(\mu, H \psi_{\mu}\right)$ given by (26) Is equivalent to the same tasks for the set of $\left(\lambda, L \phi_{\lambda}\right)$ given by (21). The relevance of the problems and methods investigated in the previous sections is thus stated for the vibration monitoring problem.

Before describing the experiments which have been done for validating our approach for the detection and diagnosis problems, let us recall that, for the identification problem $i$ using (3) and (26), strong results have been obtained from both theoretical and experimental points of view. Several known identification methods (instrumental variable, balanced realization, Ho-Kalman) have been shown to lead to consistent estimates $\hat{A}_{i}$ in (3), even with a nonstationary excitation (fast changes in the geometry of the excitation are allowed). See (Benveniste and Fuchs, 1985). From the experimental point of view, Prevosto et al. (1983) have shown that these methods are more convenient than classical Fourier methods for the identification of high 
order modes, and they investigated the stability of the identified modes with respect to the waves height and the swell direction. Other numerical results for the 1dentification problem may be found in (Prevosto, 1982) and (Prevosto et al., 1982).

\section{The diagnoses to be investigated}

With respect to the diagnosis problem statement $11 i$ which was given in the paragraph IV.1., our purpose is here more restrictive. The diagnoses we will consider are only in terms of the vibrating characteristics $\left(\lambda, L \phi_{\lambda}\right)$, rather than in terms of the physical parameters $M, C, K$. This last point is currently under study, and has not been addressed before because it is a (highly) ill-conditionned problem: in practice, the number of sensors $r$ is between 2 and 5 , and the size of the $M, C, K$ matrices 1 s several hundreds. Notice that Isermann (1985) also assumes for diagnosis that the process coefficients of interest may be uniquely determined from the model parameters at hand. Therefore, we consider only the problems described in section III, and present results concerning some Jacoblans (19) of Interest for monitoring the vibrating or modal characteristics $(\Delta, L \Phi)(21)$. The key point is the computation of convenient differentiations of equation (3), which may be written equivalently :

$$
\theta^{T} \theta_{P}(H, F)=\sum_{j=0}^{P} A_{j} H F^{D-j}=0
$$

where $A_{0}=-I_{\Gamma}$. After the change of basis defined by (27), we get :

$$
\theta^{\top} \theta_{p}\left(L \Phi, \theta^{\Delta}\right)=\sum_{j=0}^{p} A_{j} L \Phi e^{(p-j) \Delta}=0
$$


$-21-$

where

$$
\theta_{p}\left(L \Phi, e^{\Delta}\right)=\left(\begin{array}{l}
L \Phi \\
L \Phi_{e}{ }^{\Delta} \\
\vdots \\
L \Phi e \\
(p-1) \Delta
\end{array}\right)
$$

Then, differentiating (29) results in :

$$
\begin{aligned}
d \theta^{T} \cdot \sigma_{p}\left(L \Phi, e^{\Delta}\right)= & -\sum_{j=0}^{p} A_{j} L d\left(\Phi e^{(p-j) \Delta}\right) \\
& -\sum_{j=0}^{p} A_{j} L d \Phi e^{(p-j) \Delta} \\
& -\sum_{j=0}^{p-1}(p-j) A_{j} \cdot L \Phi \cdot e^{(p-j) \Delta} \cdot d \Delta .
\end{aligned}
$$

The corresponding equation for $d(\Theta)$, where $(\Theta)=\operatorname{col}\left(\theta^{\top}\right)$ was introduced in section II.1, is then :

$$
\begin{aligned}
\left(\theta_{p}\left(L \Phi, e^{\Delta}\right)^{\top} \otimes I_{r}\right) d(\Theta)= & -\sum_{j=0}^{p}\left(e^{(p-j) \Delta} \otimes A_{j} L\right) \operatorname{col}(d \Phi) \\
& -\sum_{j=0}^{p-1}\left(I_{m} \otimes(p-j) A_{j} L \Phi e^{(p-j) \Delta}\right) \operatorname{col}(d \Delta) .
\end{aligned}
$$

(30)

After some computations which may be found in Appendix $B$, we get :

$$
\left(\theta_{p}^{* T} \otimes I_{r}\right) d \Theta\left(\begin{array}{cc}
J^{\psi} & 0 \\
0 & \bar{J}_{\psi}
\end{array}\right)\left(\begin{array}{l}
d \psi \\
\overline{d \psi}
\end{array}\right)+\left(\begin{array}{cc}
J_{\lambda} & 0 \\
0 & \overline{J_{\lambda}}
\end{array}\right)\left(\begin{array}{l}
\mathrm{d} \lambda \\
\overline{d \lambda}
\end{array}\right)
$$

where $\theta_{p}^{*}$ is the observability matrix in the modal basis and easily shown to be :

$$
\theta_{p}^{*}=\left(\theta_{p}\left(L \Phi, e^{\Delta}\right), \overline{\theta_{p}\left(L \Phi, e^{\Delta}\right)}\right)
$$


$-22-$

where

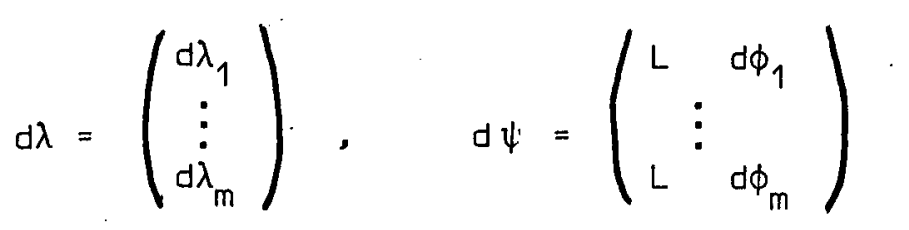

contains the variations of the observed part of the modes,

$$
\begin{aligned}
& J_{\psi}=\left(\begin{array}{ccc}
A\left(\mu_{1}\right) & & \\
& \ddots & \\
& \ddots & \\
0 & & A_{\left(\mu_{m}\right)}
\end{array}\right) \text {. } \\
& J_{\lambda}=\left(\begin{array}{ccc}
\mu_{1} A^{\prime}\left(\mu_{1}\right) L \phi_{1} & & 0 \\
& \ddots & \\
0 & & \ddots \\
\mu_{m} A^{\prime}\left(\mu_{m}\right) L \phi_{m}
\end{array}\right)
\end{aligned}
$$

with

$$
\mu_{j}=e^{\lambda j}[1 \leqslant j \leqslant m),
$$

and

$$
A(x)=-\sum_{j=0}^{p} A_{j} x^{p-j}
$$

If we use :

$$
d \psi=d \psi^{r e}+i d \psi^{i m}
$$

and

$$
d \lambda_{j}=d c_{j}+1 d \omega_{j} \quad(\sec (21))
$$


we get :

$$
\left(\begin{array}{ccc}
\theta_{p}^{*} & \otimes & I_{r}
\end{array}\right) d \Theta=\left(\begin{array}{cc}
J_{\psi} & { }^{1 J} \psi \\
- & -\bar{J} \psi \\
J_{\psi} & -J_{\psi}
\end{array}\right)\left(\begin{array}{c}
d \psi^{r e} \\
d m
\end{array}\right)+\left(\begin{array}{c}
J_{\lambda} \\
\overline{J_{\lambda}}
\end{array}\right) d c+\left(\begin{array}{c}
i J \lambda \\
-i J_{\lambda}
\end{array}\right) d \omega
$$

We may then monitor various vectors $\beta$ containing any free modal parameters among the $\Psi_{j}, c_{j}, \omega_{j}(1 \leqslant j \leqslant m)$, by select1ng the corresponding columns in the right-hand side of (32), in order to get a full column rank matrix $J_{\beta}$ such that :

$$
\left(\theta_{p}^{*} \otimes I_{r}\right) \text { d }(H)=J_{B} \quad d_{B}
$$

Using the factorization (13) written in the modal basis, it turns out that, because if (19) and (33), the sensitivity test for monitoring $\beta$ is given by (16) with :

$$
M=\left(\mathcal{U}_{N}^{* \top} \otimes I_{\Gamma}\right) \quad J_{B}
$$

where $\varphi_{N}^{*}=\Psi^{-1} \cdot \varphi_{N}\left(F, G_{S}\right)$

In the experiments which will be described in the next section, the selected vectors $\beta$ are separate vibrating modes together with the corresponding vibrating pulsation, namely :

$$
\psi_{j}^{r e}, \psi_{j}^{i m}, \omega_{j}
$$


The damping coefficients $c_{j}$ are not monitored because usually not precisely identified.

Finally, let us. mention that, as for the global test $t_{0}(16)$, theoretical investigations have been made by Rougée (1985) for computing the power of any sensitivity test of the form (16) with M given by (34). These results are also discussed in (Rougee et al., 1985) and used in (Basseville et al., 1985c).

\section{EXPERIMENTAL RESULTS}

In this section, we present results obtained from the computer implementation of the various tests presented in sections II and III, using both simulated and real vector data related to the mechanical engineering problem described in section IV. Lét us simply recall that experiments done for scalar signals are described by Basseville et al. (1985b) and lead to the conclusion that it is possible to detect and diagnose small changes $(1 \%)$ in eigenfrequencies provided that the damping coefficients are low, and that the record sizes are high enough (several thousands).

According to section IV.2, the simulation model which we used is a tied down system of 18 masses of one degree of freedom connected by springs, as shown on figure 1, with known weights, stiffness, and damping coefficients. Six dimensional signals (displacements of the masses $1,7,13,6,12,18$ ) have been generated under different hypotheses, including no change, change in mass $n^{\circ} 1$, change in the stiffness of the connection to the ground, and change in the stiffness of the connection 8-11 These physically different changes are indicated by arrows on figure 1. 
We have computed the global test $t_{0}(16)$ and nine sensitivity tests, using (34), corresponding to the nine modes of lowest order, considered together with the pulsation $\omega_{j}$ as mentionned at the end of section IV. These tests computations have been done for all the subsets of components corresponding to $r=2,3,4,6$ and to at least one observation on each of the two opposite "legs" of the structure.

For simulated as well as real data, the reference AR model $\theta_{0}$, to be used for the tests, has been computed by solving (29) where $\Phi$ and $\Delta$ contain the available eigencharacteristics (a few number for real data). The AR order $p$ is chosen to be the integer part of $2 m / r$, and not such that : $2 m / r \leqslant p<2 m / r+1$ as done for the identification task 1 of section IV. The reason for doing so is that it is intuitively more sensible to monitor the structure without introducing other modes than those which are available. The chosen number of instruments is $N=p$, for reasons which were discussed in (Basseville et al., 1985b), and therefore $t_{0}$ is computed with the aid of $(18)$.

Finally, the Jacobians $J_{\beta}$ of section IV. 3 are computed with the aid of (31), where the eigenvalues and eigenvectors which are used are the available eigencharacteristics, and not the modes associated to the computed $A_{i}$.

The results are presented on the three-dimensional figures 2 to 5 , one horizontal axis corresponding to the different sensors location and numbers, from 2 to six, from left to right, and the other horizontal axis 
corresponds to various tests, with the global test in the back and the nine sensitivity tests from the back to the front according to increasing frequencies. As pictures are drawn with automatic scaling, the maximum value is indicated. Figure 2 shows the behavior of the various tests under no change hypothesis; Figure 3 corresponds to a change (14\%) in mass 1 , figure 4 to a change $(12 \%)$ in stiffness of ground connection, figure 5 to a cancellation of connection 8-11 (which was small). These results show that some sensor locations may be very poor, in the sense that no detection is possible (nearly same value of the tests under $H_{0}$ and $H_{1}$ ), and therfore moving sensors (along legs) may be of interest. On the other hand, the visually significantly different profiles obtained on figure 3 to 5 lead to the conclusion that these types of tests contain informations which allow discrimination among physically different changes. An attempt to get direct physical diagnosis is currently under development.

\section{CONCLUSION}

We have presented an original approach for solving the two problems of detection and diagnosis of changes in the eigenstructure of nonstationary multivariable systems. This approach is based upon the use of an instrumental statistics which reduces the detection problem to a problem of change in the mean of a gaussian process with known covariance matrix. The diagnosis problem is solved in the same manner, with the aid of first order approximations relating the changes in the system parameters to be monitored to the changes in the model parameters which are used for monitoring. 
The application of this methodology to the mechanical engineering problem of vibration monitoring has been described for complex vibrating structures subject, to natural nonstationary and uncontrolled excitation. Experimental results have been presented, which show the efficiency of the proposed tests, for detecting small changes (typically a few per cent in eigenfrequencies) and for discriminating different physical changes. Our opinion is that such an approach for diagnosis ("sensitivity" method) is general enough for a possible direct use on physical parameters. This approach is currently under study. 


\section{REFERENCES}

Akaîke. H. (1974). Markovian representation of stochastic processes and its application to the analysis of autoregressive moving average processes. Ann. Inst. Stat. Math. 26, 363.

André-Obrecht. R. [1995]. Segmentation automatique du signal de parole, sans reconnaissance. Thesis, IRISA. Univ. Rennes I. France [in French].

Basseville. M.. A. Benveniste, G. Moustakides. A. Rougée [1985a]. Detection of abrupt changes in the modal characteristics of nonstationary vector signals. Proc. MTNS 85. Stockholm.

Basseville. M. [1985]. Etude des méthodes de surveillance du comportement vibratoire des structures en mer : positionnement optimal des capteurs et détection d'anomalies. IRISA Research Report $n^{\circ} 268$ [in French].

Basseville. M.. A. Benveniste, G. Moustakides (1985b). Detection and diagnosis of abrupt changes in modal characteristics of nonstationary digital signals. IEEE Trans. on Inf. Th., to appear.

Basseville. M.. A. Benveniste [Ed.] [1986]. Detection of abmut changes in signazs and dynamical systems. LNCIS n77. Springer Verlag. Berlin.

Basseville, M.. A. Benveniste, G. Moustakides, A. Rougée [1985c]. Optimal sensor location for detecting changes in dynamical behavior. To be submitted for publication. 
Benveniste. A.. J.J. Fuchs [1985]. Single sample modal identification of a nonstationary stochastic process. IEEE Trans. on aut. Cont., 30. 66.

Bohlin. T. [1977]. Analysis of EEG signals with changing spectra using a short word Kalman estimator. Math. Biosciences, 35. 221.

Chow, E.Y.. X.C. Lou, G.C. Verghese, A.S. Willsky [1986]. Redundancy relations and robust failure detection. In M. Basseville et al. [Ed.], Detection of abrupt changes insignals and dynamical systems, LNCIS n ${ }^{\circ} 77$. Springer Verlag. Berlin.

Deshayes. J.. D. Picard (1986). Off-line statistical analysis of change-point models using non parametric and likelihood methods. In M. Basseville et al. [Ed.]. Detection of abrupt changes in signals and dynamical systems, LNCIS $n^{\circ} 77$. Springer Verlag. Berlin.

Isermann. R. [1984]. Process fault detection based on modeling and estimation methods. A survey. Automatica, 20, 387.

Isermann. R. [1985]. Process fault diagnosis with parameter estimation methods. Proceedings of the 7th IFAC-IFIP-IMACS Conf. on Digital Computer Applications to Process Control, Vienna.

Mironovski. L.A. [1980]. Functional diagnosis of dynamic systems. A survey. Automation and Remote Control, 41. 1122.

Moustakides, G.. A. Benveniste [1985]. Detecting changes in the AR parameters of a nonstationary ARMA process. Stochastics, to appear.

Nikiforov. I.V. [1983). Sequential detection of abrupt changes in time series properties. Naouka, Moscow [in Russian]. 
Nikiforov. I.V. [1986]. Sequential detection of changes in stochastic systems. In M. Basseville et al. [Ed.]. Detection of abmut changes in signals and dynamical systems, LNCIS n77. Springer Verlag. Berlin.

Pau. L.F. [1981]. Failure diagnosis and performance monitoring. Marcel Dekker. New York.

Prevosto. M. [1982]. Algorithmes d'identification des caractéristiques vibratoires de structures mécaniques complexes. Thesis, IRISA. Univ. Rennes, I. France [in French].

Prevosto. M.. A. Benveniste. B. Barnouin [1982]. Modélisation et identification des caractéristiques d'une structure vibratoire : un problème de réalisation stochastique d'un grand système non-stationnaire. Research Report IRISA n $n^{\circ} 163 /$ INRIA $n^{\circ} 130$ [in english].

Prevosto. M.. B. Barnouin. C. Hoen (1983). Frequency versus time domain identification of complex structures modal shapes under natural excitation. Proceedings of the 11th IFIP Conf. on System modelling and optimization, Copenhague.

Rougée. A. (1985). Détection de changements dans les paramètres AR d'un processus ARMA vectoriel : application à la surveillance des vibrations. Thesis, IRISA. Univ. Rennes 1. France [in French].

Rougée. A.. M. Basseville. A. Benveniste. G. Moustakides (1985). Optimal robust detection of changes in the AR part of a multivariable ARMA process. Submitted for publication. 
Söderström. T.. P.G. Stoica [1980]. Instrumental variable methods for system identification. LNCIS ñ57. Springer Verlag. Berlin.

Stoica. P.G.. T. Söderström. B. Friedlander [1985]. Optimal instrumental variable estimation of the AR parameters of an ARMA process. IEEE Trans. on Aut. Cont., 30. 1066.

Willsky. A.S. [1976]. A survey of design methods for failure detection systems. Automatica, 12, 601.

Willsky. A.5. [1986]. Detection of abrupt changes in dynamic systems. In M. Basseville et al. [Ed.]. Detection of abrupt changes in signals and dynamical systems, LNCIS n77. Springer Verlag. Berlin.

Yuan. Z.D.. L. Ljung [1984]. Black-box identification of multivariable transfer functions. Asymptotic properties and optimal input design. Int. J. Control, 40. 233. 


\section{APPENDIX A}

\section{ASSUMPTIONS AND THEOREMS STATEMENT}

We here state the precise assumptions and theorems involved in our detection approach.

Let $\left(Y_{t}\right)$ be a nonstationary multivariable process having as state space representation :

$$
\left\{\begin{array}{l}
X_{t+1}=F X_{t}+V_{t+1} \\
Y_{t}=H X_{t}
\end{array}\right.
$$

where $X_{t} \in \mathbb{R}^{n}, \quad Y_{t} \in \mathbb{R}^{r}, \operatorname{cov}\left(V_{t+1}\right)=Q_{t},\left(V_{t}\right)$ is a white noise with time varying covariance matrix.

*The assumptions concerning the model (A1) are as follows :

(C1) F is full rank and asymptotically stable

(C2) there exists $k>0$ such that, for any vector $u$ and any integer $t$,

$$
E\left(u^{\top} v_{t}\right)^{4}<k\|u\|^{4}
$$

(C3) $(H, F)$ is observable, ie there exists an integer $p$ such that :

$$
\mathcal{O}_{p}(H, F) \triangleq\left(\begin{array}{l}
H \\
H F \\
\vdots \\
H F^{p-1}
\end{array}\right)
$$

is of rank $n$. 
(c4) there exists a matrixG of rank $r$ such that, for any integer $t$ :

$$
Q_{t} \geqslant G G^{\top}
$$

This last assumption, which is not a controllability condition, allows changes in the direction of the excitation.

*If the nominal model $\left(H_{0}, F_{0}\right)$ fulfills the conditions $C_{1}$ to $C 4$, then the two following theorems hold:

\section{Nonstationary low of large numbers}

$\sum_{N}(s)$ given by (10a) is asymptotically uniformly positive definite and bounded, and the estimate $\hat{\sum}_{N}(s)$ given by (10b) is consistent :

$$
\sum_{N}(s)^{-1} \hat{\Sigma}_{N}(s) \underset{s \rightarrow \infty}{\longrightarrow} I \quad \text { a.s. }
$$

under both no change hypothesis $H_{0}: \theta=\theta_{0}$, and small change hypothesis

$$
H_{1}: \theta=\theta_{0}+\frac{\delta \theta}{\sqrt{s}}
$$

\section{Nonstationary central limit theorem}

Under $H_{0}, \sum_{N}^{-1 / 2}(s) U_{N}(s) \frac{\mathscr{L}}{s \rightarrow \infty} \rightarrow Y_{(0, I)}$

where

$$
\mathcal{U}_{N}(s) \text { is given by }(9) \text {; }
$$


Under $H_{1}, \sum_{N}^{-1 / 2}(s)\left(\mathcal{U}_{N}(s)-\left(\frac{1}{s} \mathscr{H}_{\mathrm{p}, N}^{T}(s) \otimes I_{r}\right) \delta \Theta\right) \underset{s \rightarrow \infty}{\stackrel{\mathcal{L}}{\rightarrow}} \mathcal{Y}(0, I)$

*If we further assume that the following condition is fulfilled:

$$
\lim _{s \rightarrow \infty} \inf _{n}\left(\frac{1}{s} \cdot \mathscr{H}_{p, N}(s)>\sigma>0\right. \text { a.s. }
$$

where $\sigma_{n}(M)$ is the $n$-th singular value of $M$, then with (C3) we ensure the uniform minimality of the state space model (A1) to represent $\left(Y_{t}\right)$.

The assumptions (C1) to (C5) Imply the assumptions needed by Benveniste et al. (1985) for the consistency of the identification, and also imply that the factorization (13) of the Hankel matrix hold. 


\section{APPENDIX B}

\section{DIFFERENTIATION OF A MATRIX POLYMIAL}

We here give the details of the computations needed from equation (30) to equation (31), related to the Jacobians to be used for diagnosis. Rewriting (30) :

$$
\begin{aligned}
\left(\theta_{p}\left(L \phi, e^{\Delta}\right)^{\top} \otimes I_{r}\right) d \Theta(\Theta) & -\sum_{j=0}^{P}\left(e^{(p-j) \Delta} \otimes A_{j} L\right) \operatorname{col}(d \Phi) \\
& -\sum_{j=0}^{P-1}\left(I_{m} \otimes(p-j) A_{j} L \phi e^{(p-j) \Delta}\right) \operatorname{col}(d \Delta) \\
& \triangleq-\left(J_{1}+J_{2}\right)
\end{aligned}
$$

we first consider the term $\mathrm{J}_{1}$.

$$
\begin{aligned}
& -\sum_{j=0}^{p}\left[e^{(p-j) \Delta} \otimes A_{j} L\right)=-\sum_{j=0}^{p}\left(\begin{array}{cccc}
e^{(p-j) \lambda_{1}} & A_{j} L & 0 & \\
& \ddots & & \\
& & \ddots e^{(p-j) \lambda_{m}} & \\
0 & & A_{j} L
\end{array}\right) \\
& =\left(\begin{array}{cccc}
A\left(\mu_{1}\right) L & & & 0 \\
& \ddots & & \\
& & \ddots & \\
0 & & & A\left(\mu_{m}\right) L
\end{array}\right)
\end{aligned}
$$


$-37-$

where $\quad A(X)=X^{P} I_{r}-A_{1} X^{P-1}-\ldots-A_{p-1} X-A_{p}$

and $\quad \mu_{j}=e^{\lambda j}$.

Thus :

$$
J_{1}=\left(\begin{array}{cc}
A\left(\mu_{1}\right) & 0 \\
\ddots & \\
0 & \ddots \\
A\left(\mu_{m}\right)
\end{array}\right)\left(\begin{array}{c}
L d \phi_{1} \\
\vdots \\
\vdots \\
L d \phi_{m}
\end{array}\right)
$$

(BD)

On the other hand, for computing $\mathrm{J}_{2}$, we need :

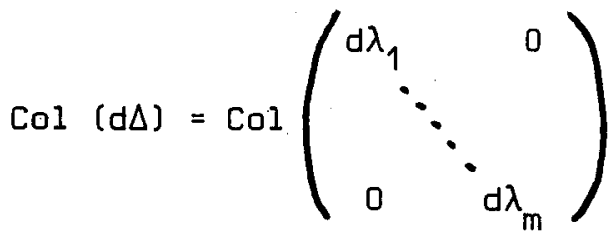

$$
\begin{aligned}
& =\left(\begin{array}{ccc}
d \lambda_{1} & & e_{1} \\
& \vdots & \\
d \lambda_{m} & & e_{m}
\end{array}\right)
\end{aligned}
$$

where $e_{j}$ is the $j$-th vector of the canonical basis of $\mathbb{R}^{m}$. Therefore :

$$
J_{2}=-\sum_{j=0}^{p-1}\left(\begin{array}{ccc}
(p-j) A_{j} L \phi e^{(p-j) \Delta} & 0 \\
0 & \ddots & \ddots \\
& & \ddots p-j) A_{j} L \phi e^{(p-j) \Delta}
\end{array}\right)\left(\begin{array}{cc}
e_{1} & 0 \\
\ddots & \ddots \\
0 & e_{m}
\end{array}\right)\left(\begin{array}{c}
d \lambda_{1} \\
\vdots \\
d \lambda_{m}
\end{array}\right)
$$


$-38-$

$$
\begin{aligned}
& =-\sum_{j=0}^{p-1}\left(\begin{array}{ccc}
(p-j) A_{j} L \phi_{1} e^{(p-j) \lambda_{1}} & 0 & \\
\ddots & \ddots & \\
0 & & (p-j) A_{j} L \phi_{m} e^{(p-j) \lambda_{m}}
\end{array}\right)\left(\begin{array}{c}
d \lambda_{1} \\
\vdots \\
d \lambda_{m}
\end{array}\right) \\
& =\left(\begin{array}{ccc}
\mu_{1} A^{\prime}\left(\mu_{1}\right) & L \phi_{1} & 0 \\
& \ddots & \\
0 & & \mu_{m} A^{\prime}\left(\mu_{m}\right) L \phi_{m}
\end{array}\right)\left(\begin{array}{c}
d \lambda_{1} \\
\vdots \\
d \lambda_{m}
\end{array}\right)
\end{aligned}
$$

(By)

where $A^{\prime}(x)=p x^{p-\vdots} I_{r}-(p-i) A_{1} x^{p-2}-\ldots-A_{p-1}$.

Including (B2) and (B3) into (B1), we get :

$$
\left(\theta_{p}\left(L \phi, e^{\Delta^{\top}}\right)^{\top} \otimes I_{r}\right) d \Theta=J_{\psi} d_{\psi}+J_{\lambda} d \lambda
$$

where $\mathrm{J}_{\psi}, \mathrm{J}_{\lambda}, d \psi, d \lambda$ are defined below formula (31).

Finally, using the observability matrix in the modal basis :

$$
\theta_{p}^{*}=\theta_{p}(H, F) \Psi
$$

where $\Psi$ is defined in (27), we get (31). 


\section{FIGURE CAPTION}

Figure 1 : Simulation system

Figure 2 : The tests under $\mathrm{H}_{0}$

Figure 3 : A change in mass 1

Figure 4 : A chenge in the stiffness of the connection to the ground

Figure 5 : A change in the connection 8-11. 


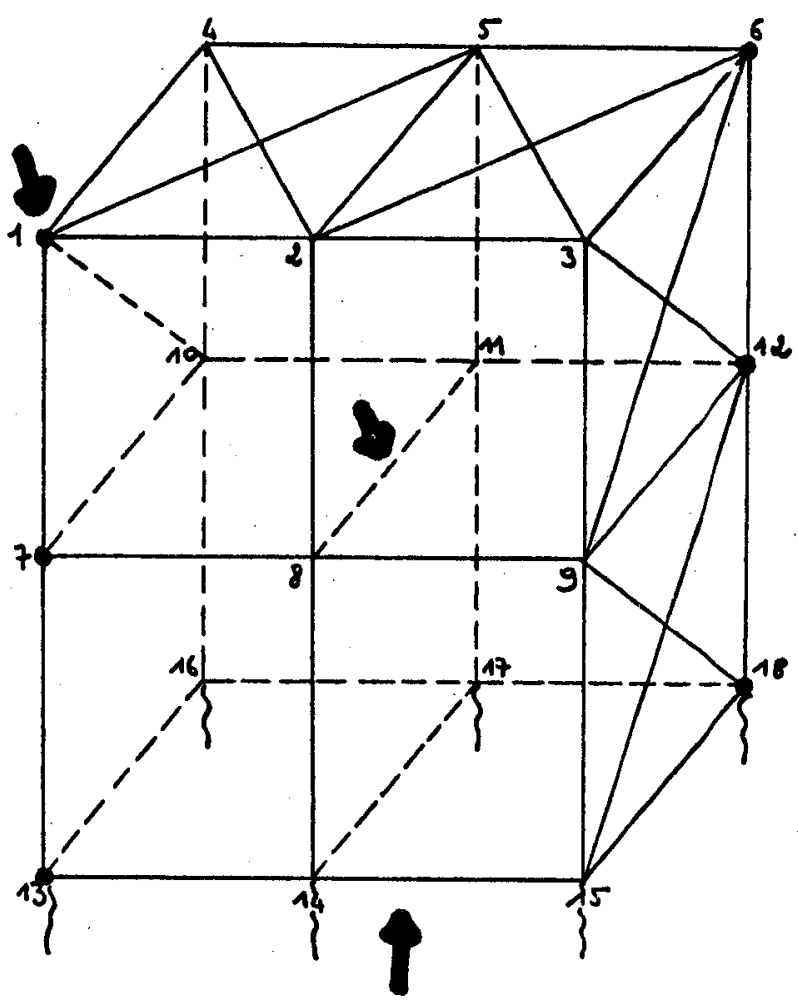

Figure 1: Simulation system 


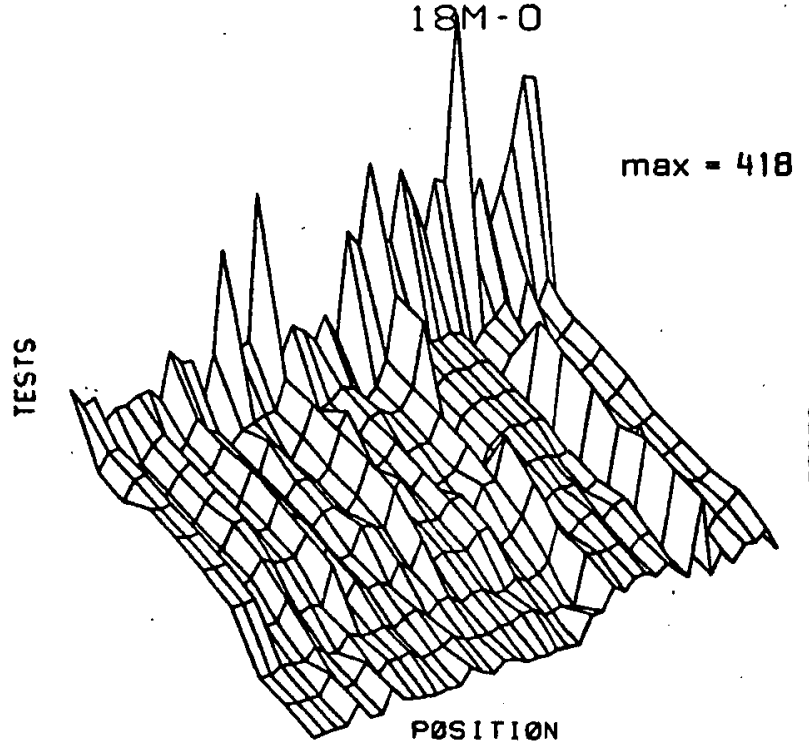

Figure 2: T ests under $\mathrm{H}_{0}$

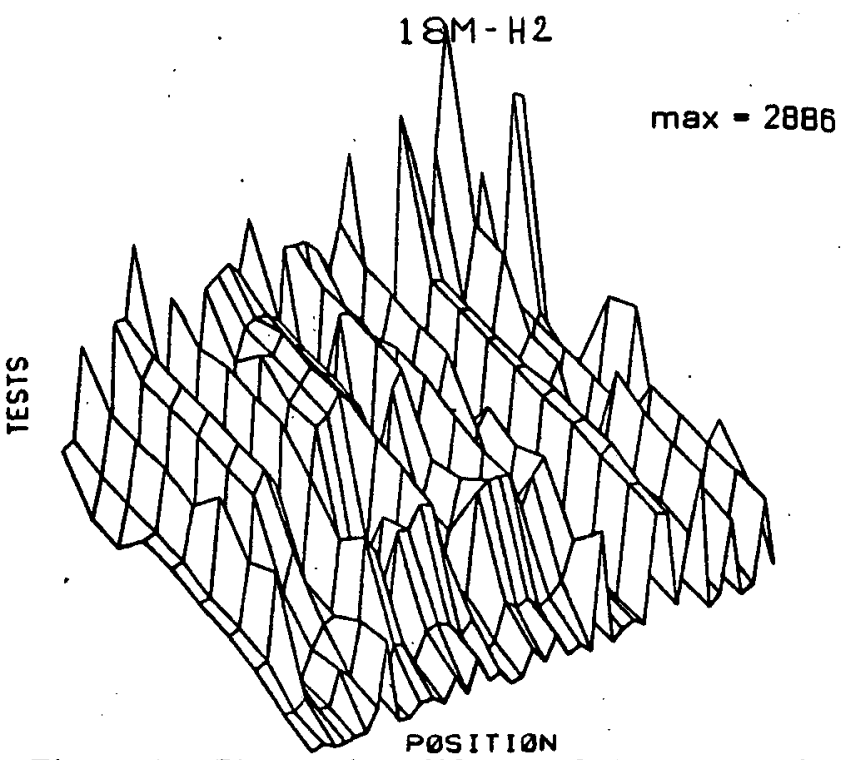

Figure 4 : Change in stiffness of the connection to the ground

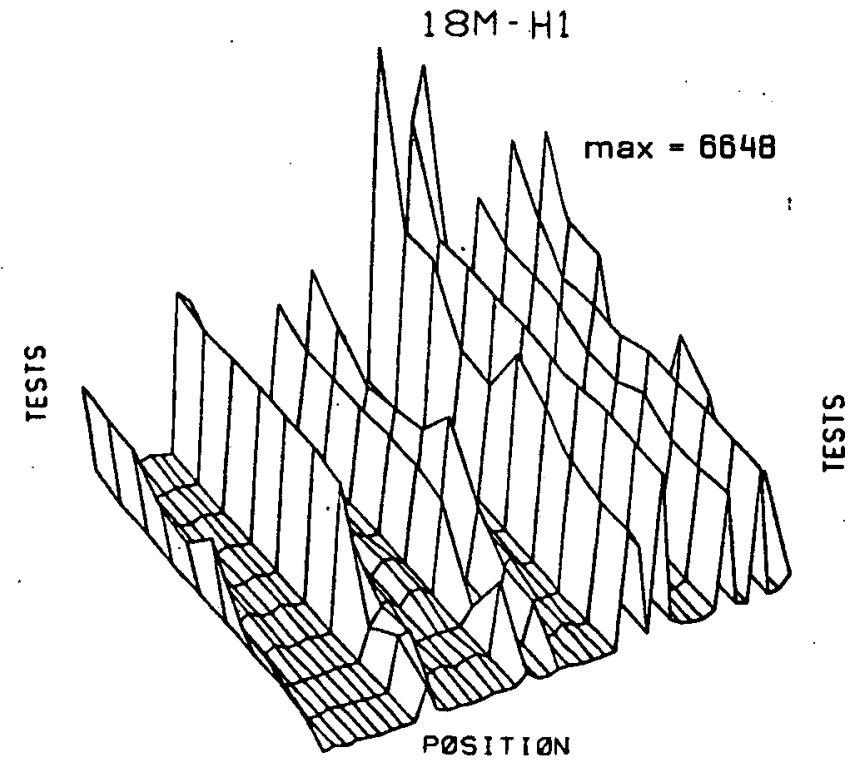

Figure 3 : Change in mass 1

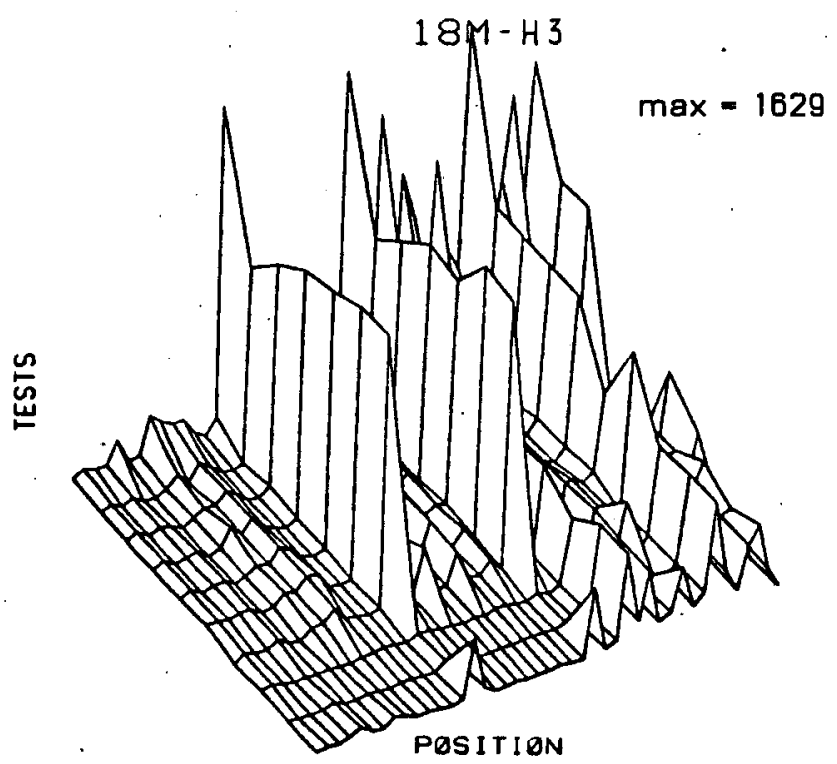

Figure 5 : Change in stiffness of the connection 8-11

Imprimé en France

par

l'Institut National de Recherche en Informatique et en Automatique 
